\title{
Coexistence of genital lichen sclerosus and genital warts
}

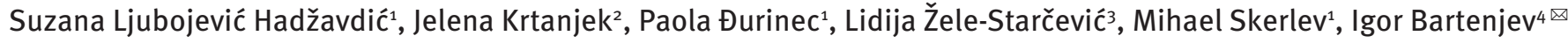

\begin{abstract}
Lichen sclerosus (LS) is a chronic, inflammatory, mucocutaneous disorder of the genital and extragenital skin. Anogenital warts are benign proliferative lesions caused by human papillomavirus (HPV), which is found in $>95 \%$ of lesions. We present two cases of the coexistence of LS and genital warts: one patient with and one without a previous history of genital warts. According to our knowledge and a literature search, only a few cases of the coexistence of LS and genital warts have been reported.
\end{abstract}

Keywords: genital lichen sclerosus, genital warts, HPV, local corticosteroids

Received: 7 May 2018 | Returned for modification: 1 June 2018 | Accepted: 5 June 2018

\section{Introduction}

Lichen sclerosus (LS) is a chronic, inflammatory, mucocutaneous disorder of the genital and extragenital skin. Itching is the main complaint in genital LS in women, and sexual and urinary dysfunction in men (1). Anogenital warts are benign proliferative lesions caused by human papillomavirus (HPV) types 6 and 11, found in $>95 \%$ of lesions $(2,3)$. HPV infection is the most prevalent sexually transmitted genital infection. The lifetime risk of infection is around 75\% (4), and it is correlated with sexual behavior (5).

The etiology of genital LS is unknown. Many factors are implicated as possible causative agents: hormonal factors, autoimmunity, HLA-DQ7 association, specific local immune reaction, trauma (Koebner phenomenon), and infections $(1,6)$. There are no clear data showing that infections-namely, those caused by Borrelia burgdorferi, Epstein-Barr virus, HPV, and hepatitis Care causative agents for genital LS $(1,6)$.

\section{Case reports}

\section{Case 1}

A 32-year-old healthy man presented with tightening of the foreskin, itching, narrowing of the urinary stream, bleeding during urination, dyspareunia, and occasional tearing of the prepuce and frenulum during sexual intercourse. Two years before the visit he was treated for genital warts with cryotherapy. On clinical examination, a pearly white, smooth area of the glans of the penis and coronal sulcus together with intraurethral papillomatous lesions at the external urethral meatus were observed (Fig. 1). Biopsy showed histological signs suggestive of condyloma acuminatum without dysplasia. HPV detection using polymerase chain reaction (PCR) of the verrucous lesion was positive for both high-risk (types 16, 18, 31, 35) and low-risk (types 6, 11, 34) HPV types, whereas HPV detection of the sclerotic lesion was positive for low-risk types (types 6, 11) and negative for high-risk types. Urethral swabs for sexually transmitted infections (STIs) and serology for syphilis, HIV, and hepatitis were negative. Initially the patient was treated twice with electrocautery for intraurethral warts. After the condyloma cleared, clobetasol propionate $0.05 \%$ ointment was applied once a day for 3 months. The frequency of local corticosteroid application was then reduced to every other day for 1 month, twice a week for another month, and finally once a week for a month. The patient was advised to stop corticosteroid treatment if the genital warts reappeared. Intraurethral condylomas reoccurred 6 months and then again 8 months after LS had cleared, and they were again treated with electrocautery. The patient has remained in follow-up for 2 years.

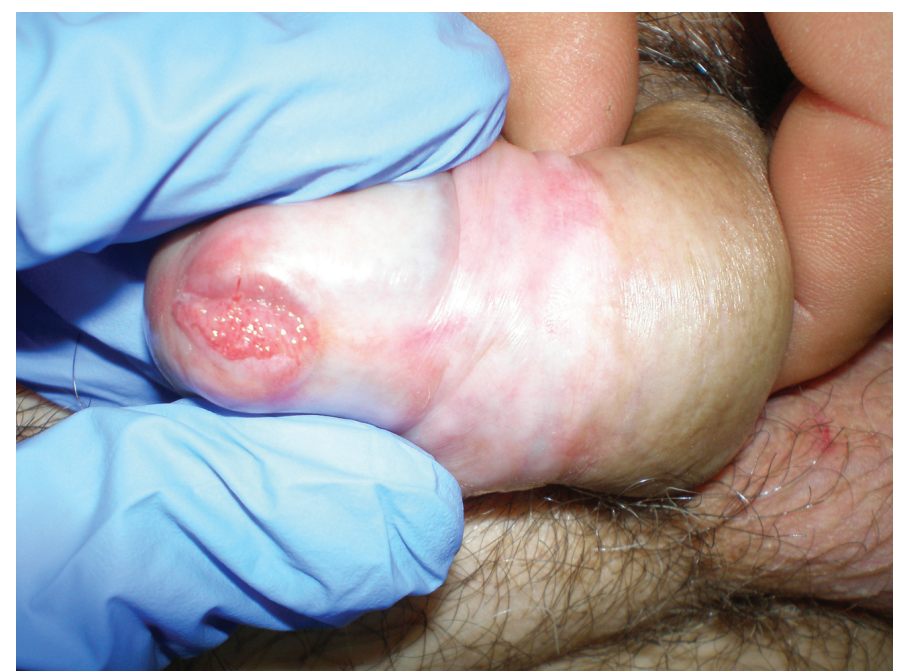

Figure 1 | Lichen sclerosus of the penis and coronal sulcus together with intraurethral papillomatous lesions at the external urethral meatus.

\section{Case 2}

A 56-year-old man with no history of genital warts and no history of other STIs presented to us with an 8-month history of penile erythema, tightening of the foreskin, ecchymoses, meatal stenosis, and dyspareunia (Fig. 2). He claimed that he had been in a monogamous relationship with his wife for over 30 years. Biopsy was consistent with LS, and he was prescribed treatment with clobetasol propionate $0.05 \%$ ointment two times a day for a month, then once a day until the follow-up visit scheduled for 3 months after the initial visit. Eight weeks after the LS therapy had been initiated, a verrucous lesion appeared on the glans of the penis (Fig. 3). A biopsy of the lesion showed condyloma acuminatum, and PCR was 
positive for HPV types 6 and 11. HPV detection of the sclerotic lesion was negative for both high-risk and low-risk HPV types. Tests for other STIs were negative. Electrocautery was performed three times and cryotherapy two times. After each condyloma had cleared, clobetasol propionate $0.05 \%$ ointment was applied once a day for 3 months, and then the frequency of application was gradually reduced. The patient was advised to stop corticosteroid treatment if genital warts reappeared. Because of frequent genital wart recurrence, imiquimod $5 \%$ cream for 14 weeks together with clobetasol propionate $0.05 \%$ ointment were applied. The duration of the treatment was 1 year. Neither visible warts nor LS lesions were observed at 2 years' follow-up.

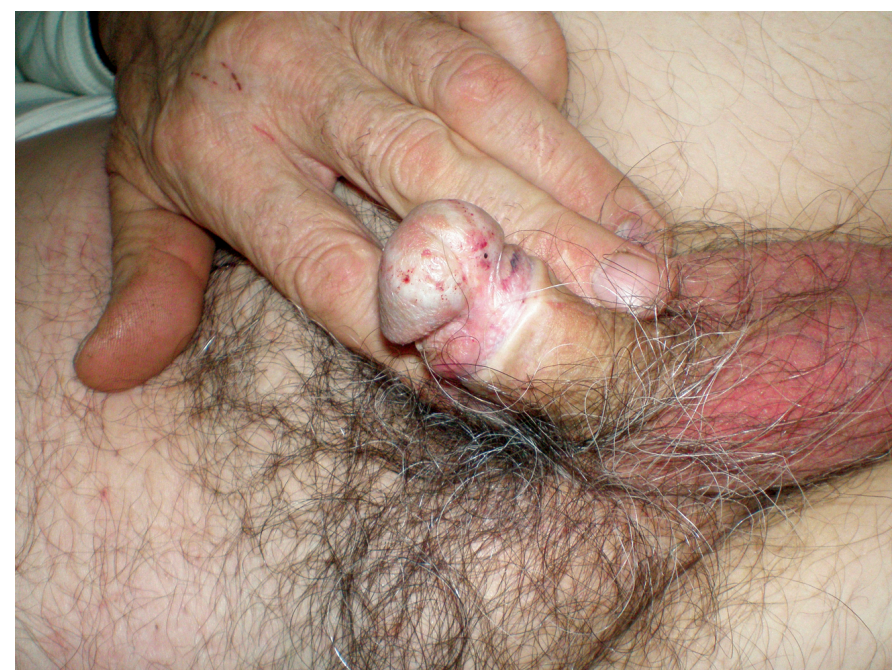

Figure 2 | Lichen sclerosus of the penis presenting with a pearly white, smooth area, ecchymoses, and meatal stenosis.

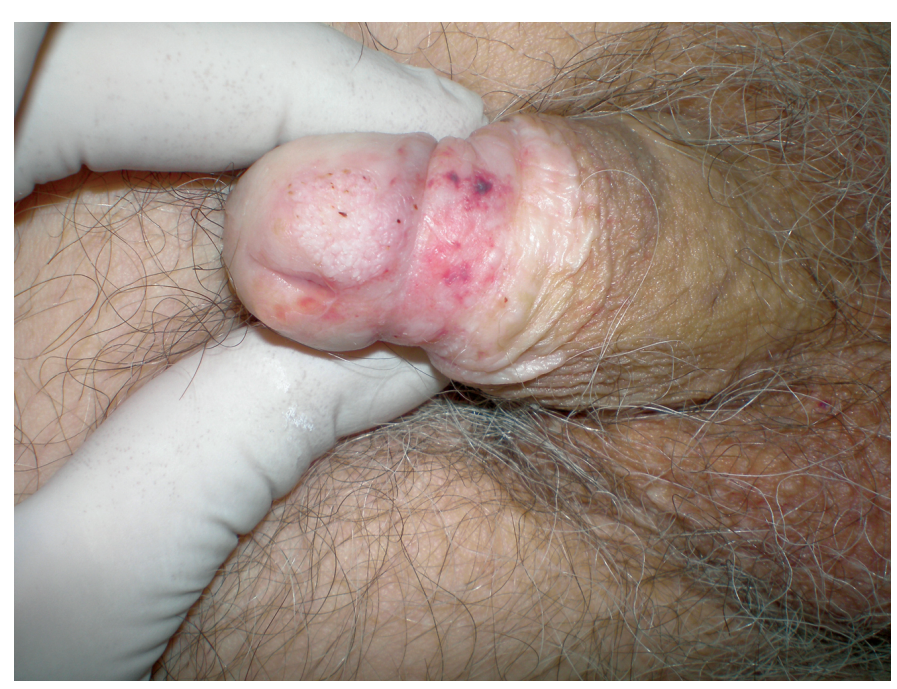

Figure 3 | Lichen sclerosus of the penis and a verrucous lesion on the glans of the penis.

\section{References}

1. Kirtschig G, Becker K, Günthert A, Jasaitiene D, Cooper S, Chi CC, et al. Evidencebased $\left(\mathrm{S}_{3}\right)$ guideline on (anogenital) lichen sclerosus. J Eur Acad Dermatol Venereol. 2015;29:e1-43.

2. Lacey CJ, Woodhall SC, Wikstrom A, Ross J. 2012 European guideline for the management of anogenital warts. J Eur Acad Dermatol Venereol. 2013;27:e263-70.

3. Brown DR, Schroeder JM, Bryan JM, Stoler MH, Fife KH. Detection of multiple human papillomavirus types in condylomata acuminata lesions from otherwise healthy and immunosuppressed patients. J Clin Microbiol. 1999;37:3316-22.

\section{Discussion}

HPV infection has been implicated as a causative agent of genital LS $(1,6)$. The median prevalence of HPV in genital LS is $22 \%$ (6). One could expect a much higher prevalence of HPV in genital LS because the lifetime prevalence of HPV is high (4). There are some facts that contradict the association of genital LS and HPV as a causative agent, such as the fact that genital LS is never seen in sexual partners; moreover, despite widespread immunization against HPV, there is no decline in the genital LS incidence, and so it can be concluded that there is a lack of clinical correlation of LS with HPV infection (6-8). Von Krogh et al. found that $32 \%$ of men afflicted with LS revealed a positive history of penile warts (7). HPV reactivation seemed to increase after local corticosteroid therapy. Reactivation of a latent HPV infection may be evoked following topical corticosteroid therapy because of an altered immune response $(6,7)$.

Both of our patients claimed to have been monogamous, without any history of genital skin diseases. The first patient had a history of condylomata 2 years prior to the LS development, but without LS treatment. The second patient did not have a history of genital warts, and a verrucous lesion appeared after local corticosteroid therapy of LS. The possible trigger factor in the first case could have been the previous treatment with cryotherapy, which could have caused a trauma (the Koebner phenomenon). In the second case, local corticosteroid, due to an altered immune response, reactivated a latent HPV infection that had probably been acquired many years prior. Imiquimod can be an effective choice for the treatment of LS (6) associated with HPV (9), but it can also provoke the development of LS (10). In our patient, imiquimod therapy in combination with local corticosteroid therapy resulted in the regression of both genital warts and an LS lesion. HPV tests of atrophic genital lesions are frequently negative. That could be explained by the fact that HPV could trigger an immune response giving rise to LS and yet be cleared by the time of biopsy (6), or that HPV can reside focally and an HPV test can be negative if the biopsy was taken in an area without HPV (6).

\section{Conclusion}

Patients with genital LS and HPV infection should be closely monitored for HPV-associated lesions following therapy with local corticosteroids. We suggest follow-up visits up to 2 years after clinical remission.

4. Walhart T. Human papillomavirus biology, pathogenesis, and potential for drug discovery: a literature review for HIV nurse clinical scientists. J Assoc Nurses AIDS Care. 2015;26:693-702.

5. Ebrahimi A, Moradi MR, Rezaei M, Kavoussi H, Madani H, Mohammadamini K, et al. Comparison of the risk factors and HPV types in males with anogenital warts with and without involvement of the urethral meatus in western Iran. Acta Dermatovenerol APA. 2017;26:55-8. 
6. Hald AK, Blaakaer J. The possible role of human papillomavirus infection in the development of lichen sclerosus. Int J Dermatol. 2018;57:139-46.

7. von Krogh G, Dahlman-Ghozlan K, Syrjänen S. Potential human papillomavirus reactivation following topical corticosteroid therapy of genital lichen sclerosus and erosive lichen planus. J Eur Acad Dermatol Venereol. 2002;16:130-3.

8. Edmonds E, Barton G, Buisson S, Francis N, Gotch F, Game L, et al. Gene expression profiling in male genital lichen sclerosus. Int J Exp Pathol. 2011;92:320-5.
9. Peiranoa RV, Dibarraza GA, Vaza JP, Domíngueza VG. Human papillomavirus in fection in vulvar lesions of lichen sclherosus et atrophicus. Study by immunohistochemistry and in situ hibridization. Actas Dermosifiliogr. 2002;92:389-92. 10. O'Mahony C, Yesudian PD, Stanley M. Imiquimod use in the genital area and development of lichen sclerosus and lichen planus. Int J STD AIDS. 2010;21:219-21. 